\title{
New APNT Ranging Signals as an Opportunity for Rationalizing Ground Infrastructure
}

\author{
Giuseppe Battista, Rachit Kumar, Okuary Osechas and Boubeker Belabbas \\ German Aerospace Center (DLR), Institute of Communications and Navigation, \\ Oberpfaffenhofen, Germany, Email: Giuseppe.Battista@dlr.de
}

\begin{abstract}
ICAO Annex 10 - Attachment H provides a guidance for a rationalization of conventional radio navigation aids to support Performance-Based navigation. An optimization of terrestrial navigation infrastructure, which includes a rationalization effort and coordinated evolution, is necessary to maintain a sufficient level of safety and operations in case of GNSS outage. This is an opportunity to introduce new signals with better ranging performance. The hybridization of new systems with legacy DME enabling a fully backup navigation system. The hybrid sytems make possible to decommission older radio navigation solutions. In this paper we present our assessment tool: marginal benefit. It is the ratio between potential DME to be decommissioned and the number of new signals deployed.
\end{abstract}

\section{Introduction}

In last decades, the shift from facility-related navigation to coordinate-based navigation was possible thanks to the introduction of Perfomance-Based navigation $(\mathrm{PBN})$ requirements. The performancebased navigation increased the flexibility to design routes and procedures according to the operational specifications, enabling a rapid increase of civil air traffic [1]. The most suitable system to fulfill PBN requirements is the Global Navigation Satellite Systems (GNSS). GNSS navigation, compared with conventional radio navigation, is safer and efficient. Although, the existing satellite-based navigation infrastructure is able to serve several demand levels, it leaves concerns for its disruptions. According ICAO Annex 10 - Attachment $\mathrm{H}$ [2], the role of terrestrial navigation aids should be re-visioned to support performance-based navigation requirements in en-route and terminal airspace, when the GNSS is not available.

A rationalization of terrestrial infrastructure is necessary to maintain an adequate level of operations in case of GNSS unavailability (jamming, spoofing, catastrophic event). Moreover, an update of terrestrial aids may also enable PBN operations for users not equipped with GNSS [2]. The ICAO strategy is a guidance to States to enable PBN navigation in any condition taking account of economic, operational and technical issues.

A short-term APNT infrastructure is based on Distance Measuring Equipment (DME) that fully support PBN operations based on RNP 1.0 navigation specifications, with an adequate network [3]. As shown in [4] and [5] there are three key limitations of current DME-based navigation network:

- lack of robustness in providing RNP.

- limited capacity in handling traffic

- gaps in providing coverage at low altitudes.

In [4], we assessed the robustness of DME network with leave-one-out fashion analysis: in order to account the ability to cope with dropped or faulted measurements. This analysis is similar to Receiver Autonoumous Integrity Monitoring (RAIM) commonly used in GNSS applications. The figures 1(a) and 1(b), show the result of leave-one-out analysis for current DME infrastructure. The figures point out the fact that less than $20 \%$ of airspace volume is supported by RNP 1.0.

Therefore, the legacy DME network needs to be complemented, we take this as an opportunity to introduce ranging signals with a greater accuracy. In [4] we proposed a method to place the complementary stations. The hybrid solution, based on a combination of new ranging system and legacy DME, provides a robust RNP 1.0 accuracy.

The introduction of new signals also present an opportunity to remove the legacy or obsolete technologies such as: Non-Directional Beacon (NDB) and VHF Omnidirection Range (VOR) [2]. The new ranging systems can be hybridized with legacy ones 


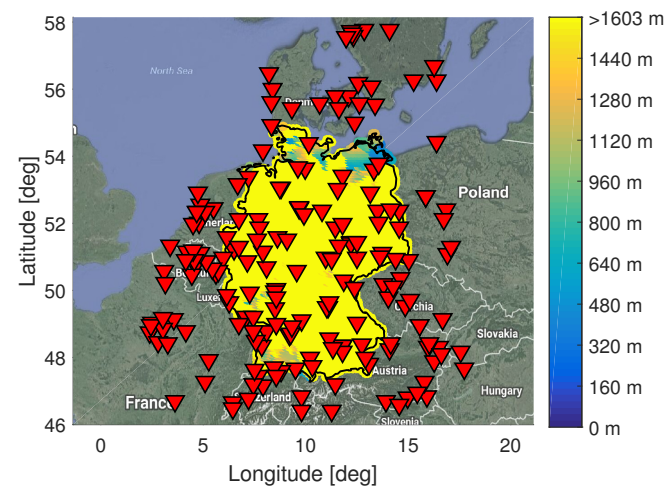

(a) Map

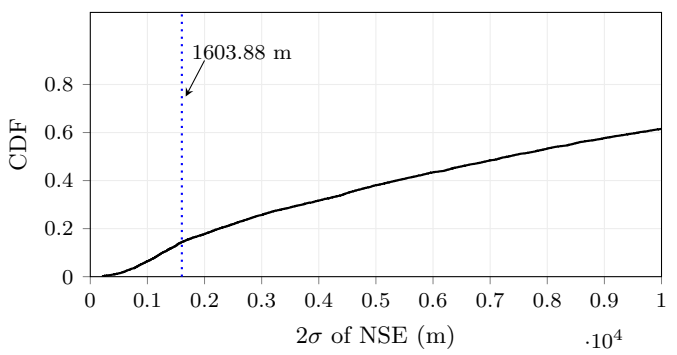

(b) $\mathrm{CDF}$

Figure 1. Leave-one-out $2 \sigma$ of NSE in Germany for DME network at FL100: map of NSE (top) and error distribution (bottom).

into one modular position system enabling a fully redundant system [6], with PBN capabilities.

\section{Scope of this work}

In this paper we present a modified version of methodology proposed in [4], which can be used to assess the impact of the new technologies at horizon on legacy DME navigation system. The deployment of new raging signals make it possible to decommission older radio navigation solutions.

In particular, our assessment tool is marginal benefit which considers newer and more efficient ground stations as an enabler for decommissioning of legacy DME. We see the opportunity to address ICAO objectives of rationalizing ground infrastructure to deploy new technologies at horizon [7] [8] [9] [10] [11].

\section{New ranging signals}

Among several candidates discussed in literature, the ranging systems considered in this paper to complement DME are:
Table I. Performance of ground-based ranging signals

\begin{tabular}{cccc}
\hline & & & \\
& Accuracy $(2 \sigma)$ & Distance & Type of ranging \\
\hline Legacy DME & $180 \mathrm{~m}$ & @ $60 \mathrm{NM}$ & Two-way \\
LDACS & $20 \mathrm{~m}$ & - & One-way \\
Mode N & $40 \mathrm{~m}$ & - & One-way \\
eDME & $80 \mathrm{~m}$ & - & One-way \\
\hline
\end{tabular}

- L-Band Digital Aeronautical Communications Systems (LDACS) [7];

- Enhanced Distance Measuring Equipment (eDME) [8] [9];

- Mode N [10].

The LDACS signal has ranging capabilities with an accuracy greater than of DME $\left(2 \sigma_{R}=180 \mathrm{~m}\right.$ at $60 \mathrm{NM}$ [12]). LDACS ranging error can be modeled with a Gaussian distribution of $2 \sigma_{R}=20 \mathrm{~m}$ [7].

Regarding DME, two improvements have been discussed in literature that can be implemented for "next-generation" of DME - enhanced DME:

- Li et al. have proposed to introduce more stable oscillators to provide carrier phase capabilities. Carrier-smoothed pulse pseudorange performance are in the order of $50 \mathrm{~m} \mathrm{[8]}$;

- Kim has proposed to change the pulse shape with an improvement of ranging accuracy in presence of noise and multipath [9].

In this work we have considered to include the aforementioned modifications in a next-generation DME enhanced DME, which has a ranging error of $80 \mathrm{~m}$ at $60 \mathrm{NM}$.

The Mode N (where "N" stands for Navigation) is new concept for ground-based navigation with a system based on second survellaince radar signals. It operates using multi-lateration concept thanks to time synchronization with GNSS or local time transfer network. A preliminary testbed shows that Mode $\mathrm{N}$ has an accuracy of $2 \sigma_{R}=40 \mathrm{~m} \mathrm{[10].}$

The performance of new signals, considered in this paper, are summarized in table I.

\section{APNT Candidates}

Several complementary candidates have been discussed in the literature. Among these, in this paper we have considered: LDACS, e-DME and Mode N. In this chapter we briefly introduce these technologies 
that would be suitable to complement the existing DME network.

\section{Legacy Distance Measuring Equipment (DME)}

DME is a two-way ranging system. The distance information is computed in the aircraft by evaluating the round-trip between interrogations from an airborne transmitter and replies to those interrogation from a ground station [12]. It operates in L-band: 960-1215 MHz. The transmitting carrier frequency and receiving carrier frequency are $63 \mathrm{MHz}$ apart. The signal is Gaussian pulse-pair shaped. The time of arrival is computed by time correlation of replied pulse pairs.

The DME performance are described in [13] and [14].

The DME has RNP 1.0 accuracy capability, if supported by suitable network [3]. The standard deviation of ranging measurement error is defined by equation:

$$
\sigma_{R}^{2}=\sigma_{s i s}^{2}+\sigma_{\text {air }}^{2}
$$

where $\sigma_{s i s}$ is signal-in-space error (distance independent, $0.05 \mathrm{NM}), \sigma_{\text {air }}$ is error due to atmospheric delays $\left(\max \left(0.085,0.00125 D_{i}\right)\right)$, from (1) we get $2 \sigma_{R}=182 \mathrm{~m}$ at $68 \mathrm{NM}$ [12].

\section{Enhanced DME}

Several DME improvements are proposed in literature: [8], [9], [11]. In this paper we considered two proposed changes to improve legacy DME performance:

- Introduction of more stable oscillators to provide carrier phase capabilities [8];

- Modification of pulse shape [9].

Carrier Phase DME Capabilities: Kuangmin Li in his Ph.D. Thesis discussed the DME carrier phase recovery as enabling technology that can improve DME ranging accuracy up to $10 \mathrm{~m}$ [8]. The carrier phase tracking would require following changes:

- transponder modification: It requires a clock stability in the order of $10^{-11} \mathrm{~s} \mathrm{~s}^{-1}$, such as the stability provided by RbXO clocks [8]. Current ground station clock accuracy is in the order of $10^{-6} \mathrm{~s} \mathrm{~s}^{-1}[8]$.

- interrogator modification: The current operating principle of DME is based on time-division multiplexing to accomodate signals from multiple beacons. To implement DME carrier tracking, we need to implement a continuouslylocked frequency synthesizer for each channel to maintain the phase continuity from multiple sources [8].

Flight trails have shown that, the implementation of DME carrier phase tracking improves accuracy significantly and boosts integrity performance, without DME spectrum modification.

Pulse Shape Improvement: Kim [9], proposed an alternative pulse waveform. The alternative DME pulse is fully compliant with pulse shape specifications of ICAO [2]. Therefore, the proposed pulse shape is compatible with legacy DME ground transponders and airborne transmitter. Additionally, it has the same spectral characteristics of traditional signal (no interference for adjacent channels). The DME accuracy is improved when is implemented, in both ground and airborne side, a Smoothed Concave Hexagonal Pulse (SCP) as in Fig. 2. The baseline of this pulse has a concave shape. The narrow width of the peak, helps in multi-path mitigation. The new pulse shape can be implemented via software upgrades. The range accuracy improvement is about $37-38 \%$ with a noise of 25-40 dB SNR and about 39$42 \%$ with costrutive short distance echoes with the direct and echo amplitude ratio of 0.3-0.5 [9].

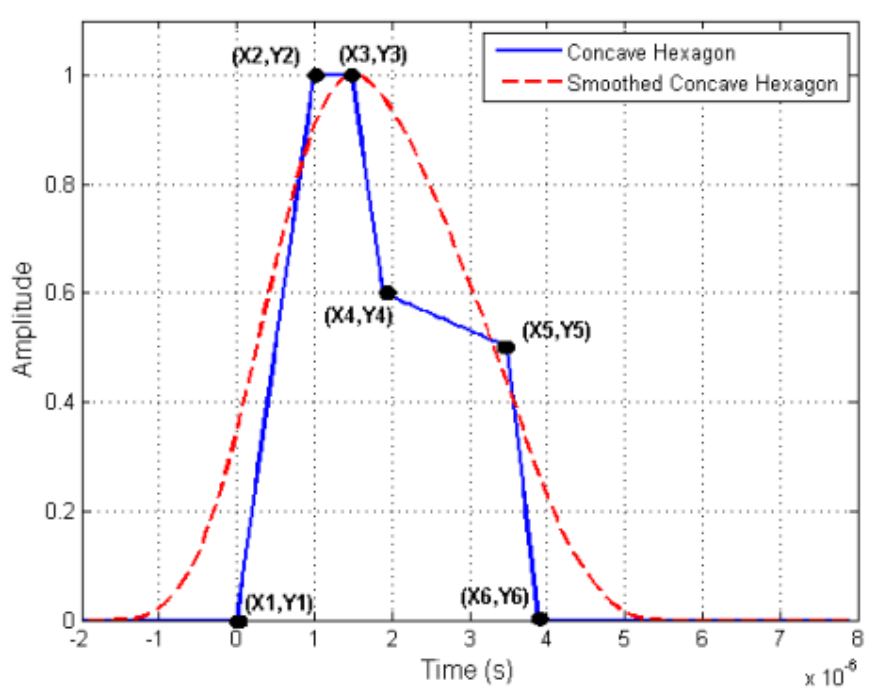

Figure 2. DME Smoothed Concave Hexagonal Pulse Shape [9].

We assumed that hypothetical enhanced DME 
has ranging error of $80 \mathrm{~m}(2 \sigma)$ at $60 \mathrm{NM}$.

\section{L-band Digital Aeronautical Communication System (LDACS)}

As shown in [7], the future aeronautical communication system LDACS has ranging capability with a ranging uncertainty of $20 \mathrm{~m}(2 \sigma)$. Airbone LDACS receiver, using trilateration or multilateration, is able to determine position peforming range with known signal source locations. It operates in L-band (960$1164 \mathrm{MHz}$ ). Each station is assigned a bandwidth of $500 \mathrm{~Hz}$. LDACS offers an interesting deploying scenario: in-lay deployment. In this case, the LDACS channel can be placed between two DME channels, spaced by $1 \mathrm{MHz}$, without interfering with them, see Fig. 3. Further, the performance of LDACS can be improved with Doppler smoothing up to 3-6 m $(2 \sigma)$ [7].

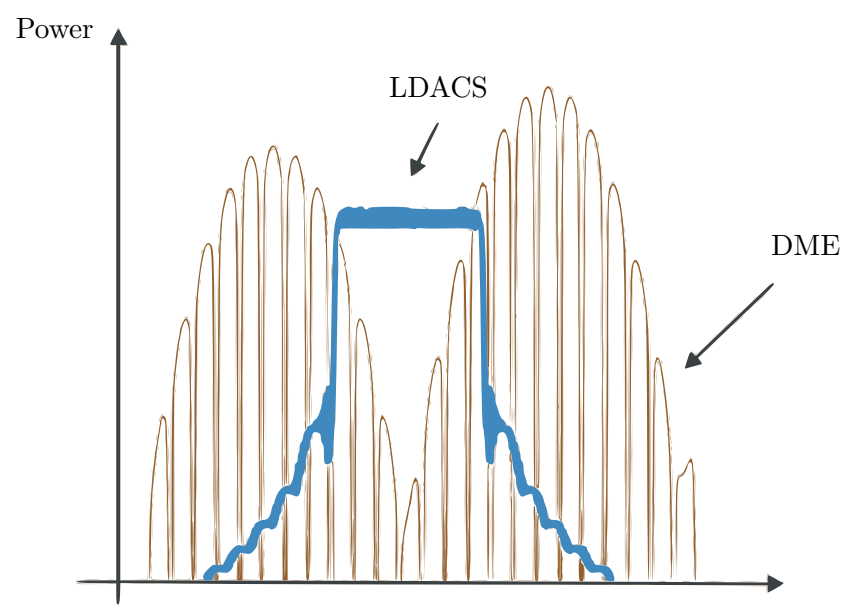

Frequency

Figure 3. In-lay option for spectral deployment of LDACS.

In this work, we assumed LDACS performance of $20 \mathrm{~m}(2 \sigma)$.

\section{Mode N}

Mode $\mathrm{N}$ is a ground-based navigation service to provide a backup navigation solution by substituting DME with a system based on SSR/Mode S signals. Mode $\mathrm{N}$ is designed to operate on a single frequency, without interfering with legacy DME. It can be deployed between 1030 and $1090 \mathrm{MHz}$, that is part of L-Band not usable by SSR due to protection by DME interference. Mode $\mathrm{N}$ is based on multilateration concept to measure the difference of transmitting time by ground stations and receiving time at airborne receiver. Mode $\mathrm{N}$ is based on SSR/Mode $\mathrm{S}$ signal and data transmissions specifications already defined in ICAO standards [10]. All ground sites are synchronized in time via: GNSS, RF time beacons and local high precision time network (for redundancy purposes). Preliminary tests have shown an accuracy of $30-50 \mathrm{~m} \mathrm{[10].}$

In this work, we assumed Mode $\mathrm{N}$ performance of $40 \mathrm{~m}(2 \sigma)$.

\section{Rationalization Strategy}

In [4], we presented a optimization method to select sites from existing aeronautical navigation/communication network as potential locations for a new technology. We have shown that a hybridization of existing DME network with a better ranging system provides a robust RNP 1.0 accuracy over German airspace. The presented method is more computationally efficient than exhaustive search to find the global optimum. A benefit of our approach is flexibility, as it can be easily adapted to place new positioning systems other than LDACS, using any existing network as potential sites.

The LDACS is an upgrade of VHF Data Link Mode 2 (VDL2), therefore the placement of LDACS sites in VHF-comm locations presents several advantages. LDACS might require similar constraints of existing communications system, e.g.: power, highspeed internet, good visibility of the sky, ease of access for maintenance purposes, etc. The density of the existing VHF-comm network sites is far greater than that of other aeronautical CNS systems. The high density provides enough flexibility to select potential LDACS sites to have a more uniform distribution in providing NSE.

Indeed, eDME can be considered as an upgrade of existing DME. The allocation of eDME in legacy DME sites might have some benefits: keep costs low, ease of upgrade in terms of hardware and software, use of existing power supply infrastructure.

Since Mode $\mathrm{N}$ is new navigation system at design stage, it might require similar characteristics of DME navigation infrastructure in terms of: power requirements, good visibility of the sky, ease of upgrade 
of hardware and software. Therefore the replacement of DME sites is convenient.

For hybrid DME-Mode $\mathrm{N}$ or hybrid DMELDACS to be fully redundant infrastructures, it would be necessary to have a time synchronization method for the synchronization of the pseudo-ranging systems.

\section{APNT Background}

\section{NSE Model: ranging and pseudoranging mea- surements}

Following notations are used:

- $\mathbf{x}$ is the user position;

- $\hat{\mathbf{x}}$ is the estimated user position;

- $b$ is the clock bias;

- $\hat{b}$ is the estimated clock bias;

- $\mathbf{s}_{i}$ is the position of $i$-th station;

- $\rho_{i}$ is the two-way range measurement for $i$-th ranging source;

- $k$ is the total number of two-way ranging sources in view;

- $N-k$ is the number of one-way ranging sources in view;

- $\rho_{k}$ is the one-way pseudorange measurement for $k$-th ranging source;

- $N$ is the total number of stations in view.

As mentioned in [15] the uncertainty of NSE is given by first two elements of covariance matrix $\mathbf{H}$

$$
\begin{aligned}
\operatorname{cov}\left[\begin{array}{l}
\Delta \mathbf{x} \\
\Delta b
\end{array}\right] & =\operatorname{cov}\left[\begin{array}{l}
\hat{\mathbf{x}} \\
\hat{b}
\end{array}\right]=\left(\mathbf{G}^{T} \mathbf{W G}\right)^{-1}=\mathbf{H} \\
\mathbf{H} & =\left[\begin{array}{cccc}
\sigma_{E}^{2} & \cdot & \cdot & \cdot \\
\cdot & \sigma_{N}^{2} & \cdot & \cdot \\
\cdot & \cdot & \sigma_{D}^{2} & \cdot \\
\cdot & \cdot & \cdot & \sigma_{T}^{2}
\end{array}\right]
\end{aligned}
$$

Given the matrix $\mathbf{H}$ in local frame, the uncertainity $(\sigma)$ of navigation system error (NSE) can be computed by equation (4):

$$
2 \sigma_{N S E}=\sqrt{H_{11}+H_{22}}=\sqrt{\sigma_{E}^{2}+\sigma_{N}^{2}}
$$

In the eq. (2), $\mathbf{G}$ is geometry matrix modified to combine ranging measurements and pseudoranging measurements:

$$
\mathbf{G}(\mathbf{x})=\left(\begin{array}{cc}
\frac{1}{\left\|\mathbf{s}_{1}-\mathbf{x}\right\|}\left(\mathbf{s}_{1}-\mathbf{x}\right)^{T} & 0 \\
\vdots & \\
\frac{1}{\left\|\mathbf{s}_{k}-\mathbf{x}\right\|}\left(\mathbf{s}_{k}-\mathbf{x}\right)^{T} & 0 \\
\frac{1}{\left\|\mathbf{s}_{k+1}-\mathbf{x}\right\|}\left(\mathbf{s}_{k+1}-\mathbf{x}\right)^{T} & -1 \\
\vdots & \\
\frac{1}{\left\|\mathbf{s}_{N}-\mathbf{x}\right\|}\left(\mathbf{s}_{N}-\mathbf{x}\right)^{T} & -1
\end{array}\right)
$$

The two-way ranging sources have index from 1 to $k$, therefore the clock components are zeros. The pseudoranging measurements have index from $k+1$ to $N$, in this case the clock components are ones. In our scenarios we assumed that all complementary systems under investigation are one-way sources.

In eq. (2), $\mathbf{W}$ is the weighting matrix:

$$
\mathbf{W}=\left(\begin{array}{ccccc}
\frac{1}{\sigma_{\rho_{1}}^{2}} & & \cdots & & 0 \\
\vdots & \ddots & & & \vdots \\
0 & & \frac{1}{\sigma_{\rho_{k}}^{2}} & & 0 \\
\vdots & & & \ddots & \vdots \\
0 & & \cdots & & \frac{1}{\sigma_{\rho_{N}}^{2}}
\end{array}\right)
$$

The weighting matrix diagonal elements are inverse of range error variance. In our assessment we use the $\mathbf{W}$ matrix for the hybrid configuration, where position solution is obtained combining pseudorange and range measurements. The diagonal elements of the weighting matrix in this case depends on the range error uncertainty of the ranging sources.

\section{Marginal Benefit}

In this paper, we introduce the concept of marginal benefit: it is the ratio between the potential legacy DME sites removables and number of newer radio signals placed. The Marginal benefit equation is (7).

$$
\text { Marginal Benefit }=M B=\frac{\text { Num. DME removed }}{\text { Num. newer sites }}
$$




\section{Rationalization Algorithm}

In [4] we presented an algorithm, where the main goal of the algorithm was to select a good subset of sites to place the complementary ranging system. It was possible to achieve an NSE low enough to support the desired RNP type, with a uniform distributions of ground stations spaced equally in azimuth direction, as in Fig. 4.

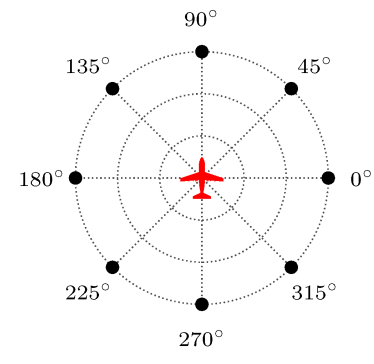

(a) 8 Ground Stations

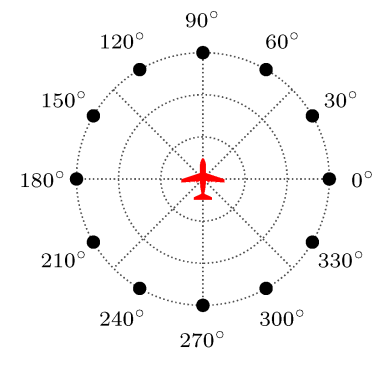

(b) 12 Ground Stations
Figure 4. Optimal geometry for ground stations with respect to the aircraft (center).

The modified version of algorithm, shown in

Fig. 5, consists in following steps:

1) pick a point $j$ on the grid as a virtual aircraft;

2) select DME and already complementary placed stations in view;

3) check if NSE is lower than the required threshold;

4) divide the ENU plane into sectors, as shown in Fig. 5;

5) compute for each region the ratio between the number of stations in the area and total number of stations in view;

6) find sites in the outer regions with lower ratio, with a cardinality of 2 ;

7) compute NSE of new hybrid configuration;

8) check if NSE is lower that the required threshold;

9) if the answer is no: increase cardinality, and go again to step 5. If the answer is yes:

(i) stop the search, before moving to next point we will check DME stations unnecessary for NSE achievement;

(ii) check the DME stations, with a cardinality of 2 and with brute force method, that if missing the NSE is always lower than the required threshold;

(iii) check again if NSE, without selected DME is lower than the required threshold. If yes increase the cardinality (until a maximum of 5) and check again the NSE;

(iv) continue with next point on the grid.

\section{Rationalization scenarios}

In this chapter we describe the various parameters and constrains implemented in rationalization algorithm, followed by the results of the algorithm in three scenarios. The three scenarios differ for ranging accuracy, we considered three complementary systems with respectively: $2 \sigma_{R 1}=20 \mathrm{~m}, 2 \sigma_{R 2}=40 \mathrm{~m}$, $2 \sigma_{R 3}=80 \mathrm{~m}$.

\section{Algorithm Input Parameters} rithm:

The following assumptions are used in algo-

- Legacy DME specifications:

- 200 NM maximum coverage distance $\left(D_{\max }\right)$, see Fig. 6;

- $1 \mathrm{deg}$ minimum elevation $\left(\alpha_{\min }\right), 50 \mathrm{deg}$ maximum elevation $\left(\alpha_{\max }\right)$. See Fig. 6;

- $2 \sigma_{R}$ range uncertainty given by eq. (1);

- Complementary ranging signals are pseudoranging systems, i.e. unlimited aircraft handling capacity. Specifications are:

- Enhanced DME:

* $D_{\max }=120 \mathrm{NM}$;

$* \alpha_{\min }=1 \mathrm{deg}, \alpha_{\max }=50 \mathrm{deg}$;

* $2 \sigma_{R}=80 \mathrm{~m}$;

* Reference network for placement: DME.

- Mode N:

* $D_{\max }=150 \mathrm{NM}$;

$* \alpha_{\min }=1 \mathrm{deg}, \alpha_{\max }=50 \mathrm{deg}$;

* $2 \sigma_{R}=40 \mathrm{~m}$;

* Reference network for placement: DME.

- LDACS:

* $D_{\max }=180 \mathrm{NM}$;

$* \alpha_{\text {min }}=1 \mathrm{deg}, \alpha_{\max }=60 \mathrm{deg}$;

* $2 \sigma_{R}=20 \mathrm{~m}$;

* Reference network for placement: VHF-comm

- Flight altitude is $3100 \mathrm{~m}$, i.e. FL100;

- 70 DME sites in Germany;

- 580 VHF-comm sites in Germany;

- Visibility analysis using Nasa's Aster Global Digital Elevation Model (DEM) with RMS between $10 \mathrm{~m}$ and $25 \mathrm{~m} \mathrm{[16];}$ 


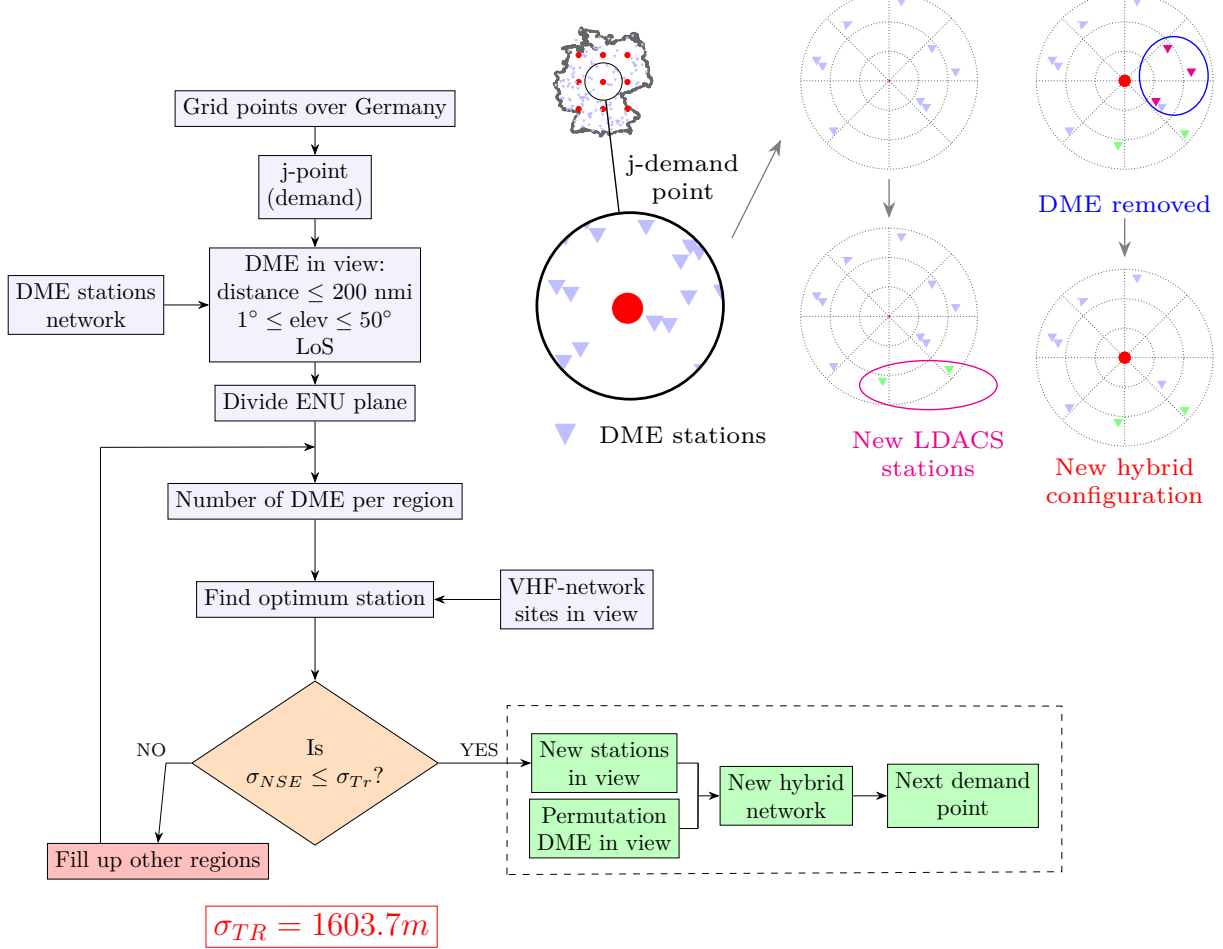

Figure 5. Algorithm to select complementary sites and remove unnecessary DME stations.

- The support grid for algorithm testing has $10 \times 10$ points, with a spacing of 1.019 deg along longitude and 0.865 deg along latitude.

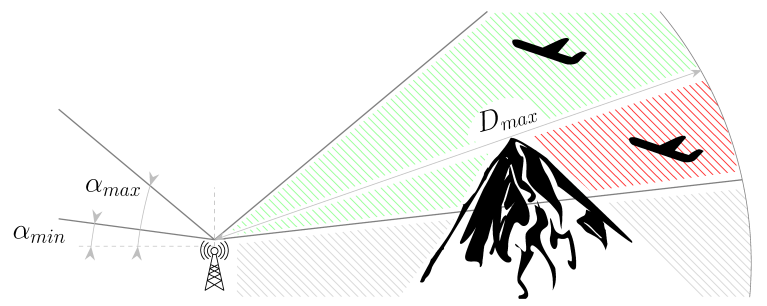

Figure 6. Representation of maximum coverage distance $\left(D_{\max }\right)$, elevation mask $\left(\alpha_{\min }-\alpha_{\max }\right)$ and line-of-sight between ground station and aircraft.

\section{Rationalization Results}

In all above cases we assessed the robustness of RNP 1.0 accuracy with leave-one-out fashion taking into account the decommissioned DMEs. Using the algorithm described earlier, we have obtained the results described in Tab. II. The results are shown in Fig. 7, 9, 8. Fig. 10 is a comparison of cumulative error distributions. Finally if fig.11, there are decom-
Table II. Results of proposed algorithm: number of stations placed and feasible DME removable

\begin{tabular}{cccc}
$\begin{array}{c}\text { Range } \\
\text { Accuracy [m] }\end{array}$ & $\begin{array}{c}\text { Stations } \\
\text { placed [-] }\end{array}$ & $\begin{array}{c}\text { DME } \\
\text { removed [-] }\end{array}$ & $\begin{array}{c}\text { Marginal } \\
\text { benefit [-] }\end{array}$ \\
\hline$\sigma_{R 1}=20$ & 26 & 48 & 1.84 \\
\hline$\sigma_{R 2}=40$ & 20 & 45 & 2.25 \\
\hline$\sigma_{R 3}=80$ & 20 & 43 & 2.15 \\
\hline
\end{tabular}

missioning profiles depending on the new deployed technology.

\section{Discussion}

In this paper, we presented a modified version of our algorithm (presented in [4]) to address ICAO rationalization strategy [2]. In the frame of strategy we presented three possible scenarios where we have deployed new signals, and at the same time it is possible to decommission legacy DME sites. As shown in the fig. 10, all scenarios are able to support robust RNP 1.0 accuracy over Germany. Interesting is the hybrid LDACS-DME case, we are very close to RNP 0.3 accuracy requirements. The fig. 11 reports the trends for marginal benefits for 


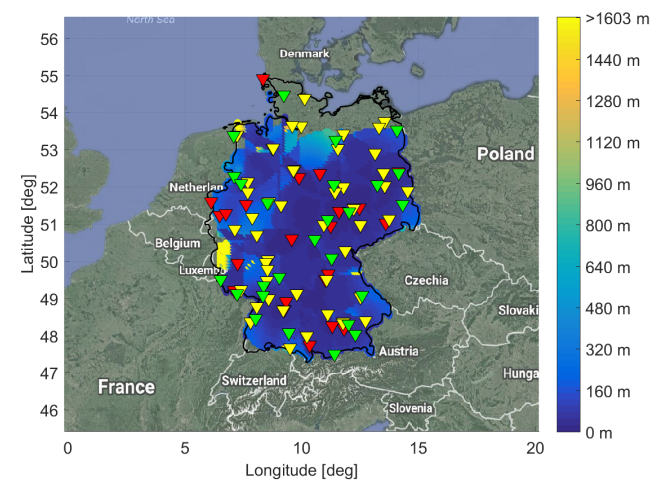

(a) Map

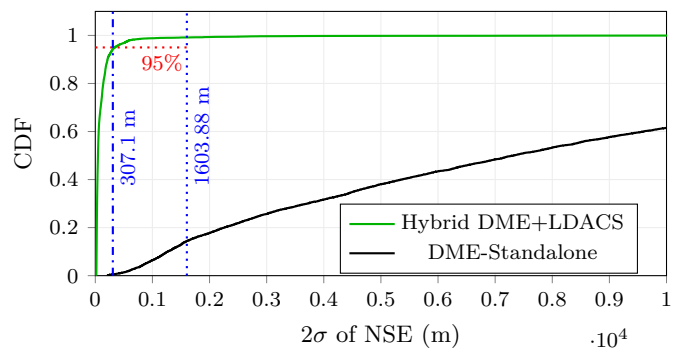

(b) $\mathrm{CDF}$

Figure 7. Leave-one-out $2 \sigma$ of NSE in Germany for hybrid network DME+LDACS at FL100: map of NSE (top) and error distribution (bottom).

all three technologies under investigation. The trends are given by the algorithm applied with different spacing of support grid. The marginal benefit is the same for hybrid scenarios with Mode $\mathrm{N}$ and DME. For the hybrid case of LDACS-DME the number of complementary stations are higher compared to hybrid case of Mode N-DME or eDME-DME. This is different from expected, as LDACS has higher ranging accuracy. A way to explain this could be the limitation in placement of LDACS ground stations, as the VHF stations are clustered. The Mode $\mathrm{N}$ and eDME has geometrical advantage of the distributed existing DME network.

In hybrid LDACS-DME network, we can observe a flat area since $48 \mathrm{DME}$ stations removed. It means that 26 LDACS are sufficient to cover the same level of operations even in lack of more DME stations.

A flat area is observable also in hybrid eDMEDME and Mode N-DME networks. In this range, approximately 20 complementary sites are able to fulfill RNP 1.0 accuracy requirements even if 45 DME stations are decommissioned.

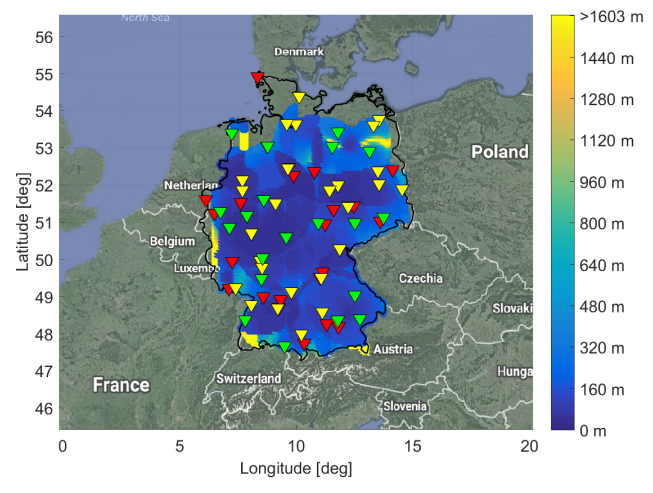

(a) Map

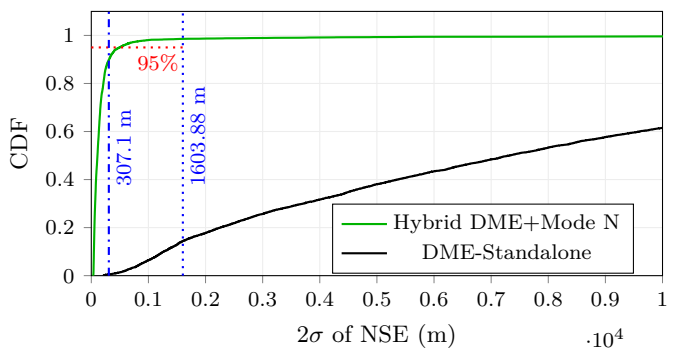

(b) $\mathrm{CDF}$

Figure 8. Leave-one-out $2 \sigma$ of NSE in Germany for hybrid network DME+eDME at FL100: map of NSE (top) and error distribution (bottom).

In the all scenarios, a blank area is observable in northern Germany. This "boundary effect" is due to the fact that we have not considered DME stations from neighboring countries. The removal of DME stations in neighboring countries, further reduces the number of visible ground station in already sparse distribution, preventing a positioning.

The algorithm presented can be adapted to address service levels other than RNP 1.0 or RNP 0.3 . It can be used also with other APNT candidates.

\section{Summary}

The proposed scenarios of hybrid navigation infrastructures guarantee high accuracy over Germany, as shown in Fig. 7, 9, 8. Although the goal was to achieve robust RNP 1.0 accuracy. In the presented scenarios, we have shown a possible rationalization of legacy DME. The ICAO Annex 10 encourages a rationalization of DME sites in areas with high density:

- In these area, the deployment of new DME stations is hard, in terms of spectrum assignment. 


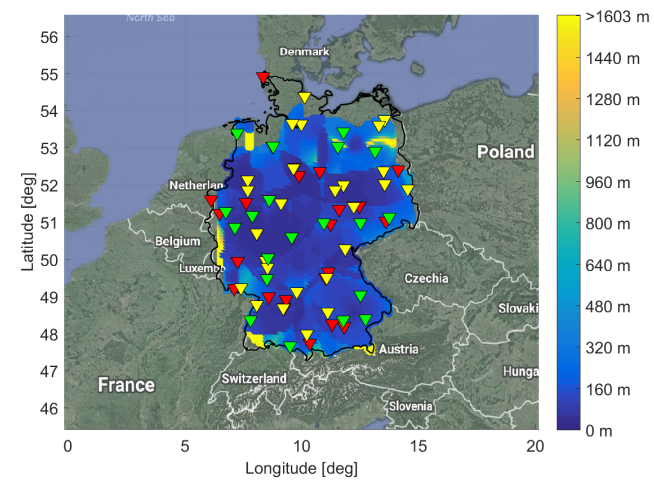

(a) Map

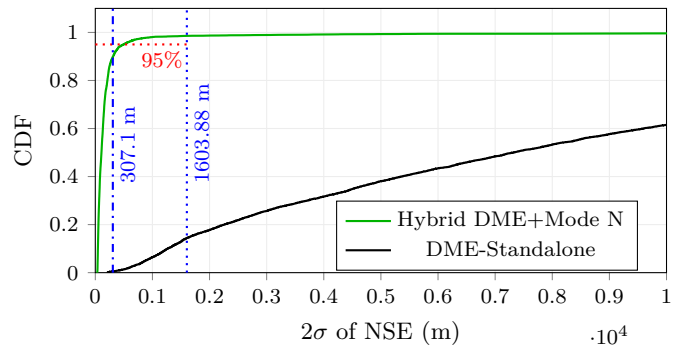

(b) $\mathrm{CDF}$

Figure 9. Leave-one-out $2 \sigma$ of NSE in Germany for hybrid network DME+Mode N at FL100: map of NSE (top) and error distribution (bottom).

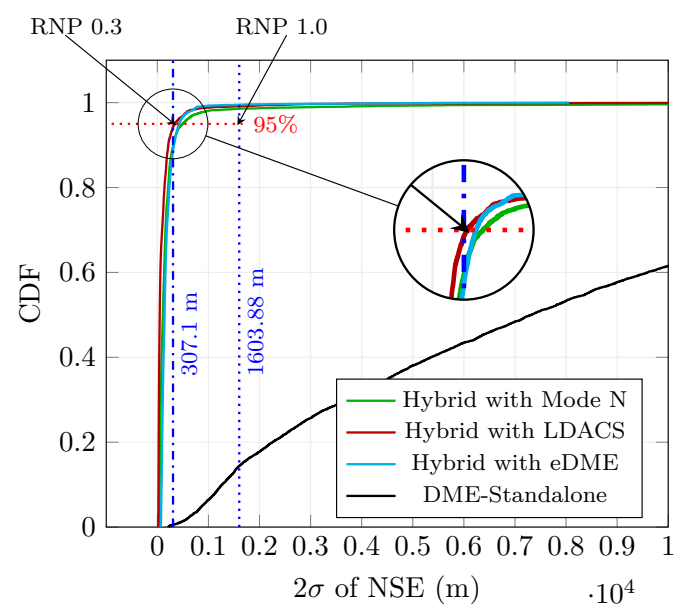

Figure 10. CDF comparison between all hybrid scenarios: LDACS-DME, eDME-DME, Mode NDME.

- At the same time, due to high power emitted by DME stations, the decommissioning of DME ground beacons might reduce interference with other radio aeronautical services operating in Lband.

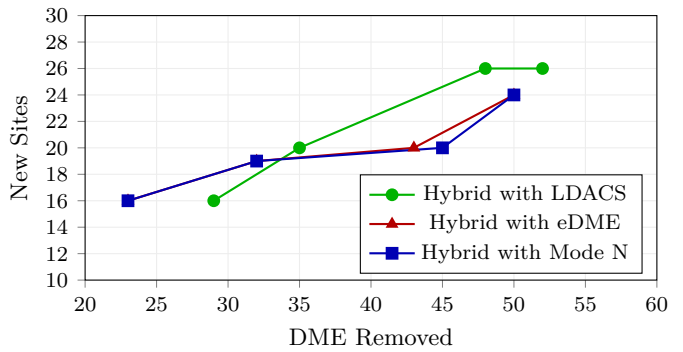

Figure 11. Marginal benefit trend for all hybrid scenarios.

We introduced an important parameter for rationalization process that is the marginal benefit. It is interesting to note that due to geometrical limitation, even though LDACS has better ranging accuracy the marginal benefit is low compared to other considered technologies in this paper. The hybrid DME-LDACS requires 26 LDACS stations in absence of $48 \mathrm{DME}$ sites. For modular Mode N-DME infrastructure, placing 20 Mode $\mathrm{N}$ stations we can remove 45 legacy DME. Regarding hybrid eDME-DME network, we can decommission 40 stations if 20 DME are upgraded to next-generation of DME - eDME.

There are still concerns about assuring integrity for all terrestrial navigation systems. An assessment of multipath propagation and detection of it, will remain topics for future works. The RAIM-like analysis that we have done might be a further step to integrity assurance. Finally, for all systems cited in this paper, it is important to implement a fault monitor on-board.

\section{References}

[1] EUROCONTROL, "Long-term Forecast - Flight Movements 2010-2030," EUROCONTROL, 96 Rue de la Fusee, B-1130 Brussels, Tech. Rep., 2010.

[2] ICAO, "Annex 10 - Aerounatical Telecommunications - Volume I Radio Navigation Aids," ICAO, 999 University Street, Montreal, Quebec, Canada, Tech. Rep., 2008.

[3] G. Berz, V. Vitan, and I. Skyrda, "Can Current DME Support PBN Operations with Integrity?" In Proceedings of the 26th International Technical Meeting of The Satellite Division of the Institute of Navigation, ION, Ed., 2013. 
[4] G. Battista, R. Kumar, E. Nossek, and O. Osechas, "Placing LDACS-based ranging sources for robust RNP 1.0 accuracy en-route," in 2017 IEEE/AIAA 36th Digital Avionics Systems Conference (DASC), 2017.

[5] R. Kumar, G. Battista, and O. Osechas, "Demandbased Placement of LDACS Ground Stations to Achieve RNP 0.3 Accuracy for APNT," in Proceedings of the 2017 International Technical Meeting of The Institute of Navigation, The Institute of Navigation, 2017, pp. 1227-1241.

[6] O. Osechas, E. Nossek, B. Belabbas, and M. Meurer, "A Modular Approach to Integrity for APNT," in 29th International Technical Meeting of The Satellite Division of the Institute of Navigation (ION GNSS+ 2016), 2016.

[7] T. Thiasiriphet, N. Schneckenburger, and M. Schnell, "Ranging with LDACS: Results from Measurement Campaign," in 2016 Integrated Communications Navigation and Surveillance (ICNS), 2016, pp. 1-18.

[8] L. Kuangmin, "Enhanced distance measuring equipment carrier phase," $\mathrm{PhD}$ thesis, Ohio University, Electrical Engineering (Engineering and Technology)., 2014.

[9] K. Euiho, "Alternative dme/n pulse shape for apnt," in 2013 IEEE/AIAA 32nd Digital Avionics Systems Conference (DASC), 2013, pp. 4D2-1-4D2-10.
[10] S. Marquard, "Mode $\mathrm{n}$ - a concept of modern navigation and apnt for aviation," in International Symposium on Precision Approach and Performance Based Navigation 2017 (ISPA), 2017.

[11] S. Lo, "Pseudolite Alternatives for Alternate Positioning, Navigation, and Timing (APNT)," FAA White Paper, August, 2012.

[12] R. J. Kelly and D. R. Cusick, Eds., Distance Mesuring Equipment and its Evolving Role in Aviation, ser. Advances in Electronics and Electron Physics. Academic Press, 1986, vol. 68.

[13] EuroCAE, Minimum performance specification for distance measuring equipment (dme/n and dme/p) (ground equipment), 1986.

[14] FAA, Performance specification distance measuring equipment faa-e-2996, 2008.

[15] Global positioning system: Signals, measurements, and performance. Ganga-Jamuna Press, 2012. [16] NASA. (Jan. 2017). ASTER NASA Digital Elevation Model, [Online]. Available: https://lpdaac. usgs.gov/.

2018 Integrated Communications Navigation and Surveillance (ICNS) Conference April 10-12, 2018 